\title{
Administering questionnaires to older people: Rigid adherence to protocol may deny and disacknowledge emotional expression
}

\begin{abstract}
This paper draws on data from a larger study conducted in care home facilities in: Seattle, USA; West Sussex and Surrey in the UK; and in the lower North Island in New Zealand. Two extracts from interactions between the researchers and an older person during the administration of The Philadelphia Geriatric Morale Scale in a care home facility in New Zealand were analysed following Houtkoop-Steenstra and using a Conversation Analysis (CA) approach. In the first extract the audio-recorded transcript was examined for events of institutional talk and rephrasing of questionnaire questions. We also examined the transcript for missed cues and the impact of closed questions when administrating questionnaires to older people living in care home facilities. We then present an extract where the researcher uses a conversational approach during the administration of the same questionnaire. We conclude that rigid adherence to interview protocols when administering questionnaires to older people who cannot complete these themselves disables the interviewer from interacting and engaging in a meaningful conversation or responding to cues that indicate distress or expressions of grief. The effect of this approach may deny and disacknowledge older persons" emotional experiences and for the older person the interview may not be a therapeutic encounter. Based on our analysis and experiences of conducting this research we support recommendations that a collaborative approach, allowing an interactional exchange between interviewer and respondent, be used when administering questionnaires to older people in care home facilities.
\end{abstract}

\section{Key Words:}

administering questionnaires; conversation analysis (CA); aged care; institutional talk; rephrasing questionnaire questions; missed cues; closed questions; disacknowledging emotional experiences

\section{Introduction/Background}

\section{Interviewing Older People and Standardized Questionnaires.}

Questionnaires are frequently used in research undertaken in aged care facilities. They are popular as methods of collecting large amounts of data quickly and efficiently.

Standardisation is emphasised in questionnaire data collection as a means of increasing the reliability and validity of the results by controlling and reducing interviewers" contributions to error (Fowler \& Mangione 1990; Schaeffer 1991; Schaeffer \& Maynard 2002). Interaction in survey interviews is supposed to be standardised, predictable, and unvaried (Fowler \& 
Mangione 1990; Schober \& Conrad, 1997; Conrad \& Schober, 2000; van „t Hof, 2006) and the goal of standardisation is to control and reduce interviewers" contributions to bias and systematic influences of interviewers on respondents (Fowler \& Mangione 1990; Schober \& Conrad, 1997; Schaeffer \& Maynard 2002).

Generally during the administration of questionnaires interviewers are expected to maintain the neutrality of the questionnaire by sticking to the rules of standardised interviewing. Strict standardisation protocols require the interviewer to: read the question exactly as worded; if the respondentes $\mathrm{s}$ answer is incomplete or inadequate then probe for clarification or elaboration in a non-directive way; record the answers without interviewere ${ }^{\text {ee }}$ discretion; and do not provide any positive or negative feedback regarding the specific content of responder ${ }^{\text {ee }}$ answers (Houtkoop-Steenstra, 2000:9). It is expected that interviewers remain interpersonally neutral and not give feedback that is evaluative; that conveys approval or disapproval to the response (Schaeffer \& Maynard 1996). When it is necessary to probe an answer, the interviewers must not ask leading questions (Fowler \& Mangione, 1990). It is argued however, that although this may be the theory of standardised interviewing, in reality this is often not the case (Suchman \& Jordan, 1990; Houtkoop-Steenstra \& Antaki, 1997;

Schober \& Conrad, 1997; Houtkoop-Steenstra, 2000; Conrad \& Schober, 2000; Schaeffer \& Maynard, 2002; Viterna \& Maynard, 2002; van „t Hof, 2006;).

\section{Rephrasing Questions and "Institutional Talk"}

Rephrasing of questionnaire questions is the most common intervention, referred to as an „interactional device ${ }^{e e}$. These are used by interviewers to generate an adequate or recordable answer if the answer given by the respondent does not follow the question (HoutkoopSteenstra, 1996). The interviewer may rephrase the question to encourage positive, face- 
protective responses, particularly in environments that are marked by „interactional troubles" (Houtkoop-Steenstra \& Antaki, 1997). Interactional troubles refers to responses that occur when asking supposedly neutral questions from an interview schedule where the aim is to deliver them in a way that worries can be anticipated and reduced and to encourage positive responses, also referred to as „,institutional talke (Houtkoop-Steenstra \& Antaki, 1997). Interviewers have been shown to use reformulation and delivery of standard and neutral questions in such a way as to pre-empt or minimise troubles in the interviewees ${ }^{\text {ee }}$ responses or to encourage positive and optimistic responses, referred to as „high-grade assessments ${ }^{\text {ee }}$ (Houtkoop-Steenstra \& Antaki, 1997; Antaki, Houtkoop-Steenstra, \& Rapley, 2000). Highgrade assessments e.g. "brilliant," "terrific," work differently from markers closing off a question using "neutral or conventionally "positive” topic-transition tokens like "right," “ok" or "right/ok then" (Antaki et al., 2000:236). Antaki et al. (2000:236) suggest that highgrade assessment sequences "claim a closure on the previous material as having been, in the circumstances, successfully completed as a section in a segmented whole”.

\section{Closed Questions vs Conversation}

It has also been noted that there is an "unresolved tension between the survey interview as an interactional event and as a neutral measurement instrument" (Suchman \& Jordan, 1990:

232). Turning the interview into an instrument disallows interaction between the interviewer and respondent (Suchman \& Jordan, 1990; Schober \& Conrad, 1997; Conrad \& Schober, 2000). However, few researchers have critically examined the use of questionnaires to collect data from older people. Isaksson et. al. (2007) carried out a small study to explicate the support given to very old people in the process of completing a research questionnaire. Twelve community dwelling people aged 90 years or older in northern Sweden completed a Resilience Scale questionnaire in a supportive face-to-face manner with the researcher. The 
researchers analysed the transcripts of audio-recorded administration of the questionnaire using content analysis. Their findings support the positions of Suchman and Jordan (1990), Schober and Conrad (1997) and Conrad and Schober (2000) in that the conversational approach to administration of the questionnaires, that is, engaging in an encouraging, explanatory, or pensive dialogue with the participants, did not detract from collecting valid and appropriate data.

\section{Missed cues}

A cue is perceived by the way the brain processes auditory, tactile and visual senses then interprets the signals and allows action (Ernst \& Bülthoff, 2004). There are different types of cues which could be verbal or nonverbal, consequently, each individual will respond and act differently to different cues and signals. By missing cues people don "et engage with each other. The patient's perspective is often expressed to the provider through emotion cues (Del Piccolo, Goss, \& Bergvik, 2006) and verbal or nonverbal cues which suggest an underlying unpleasant emotion would need clarification by a health professional (Zimmermann, Del Piccolo, \& Finset, 2007; Zimmermann et al., 2011; Del Piccolo, Mazzi, Goss, Rimondini, \& Zimmermann, 2012). Patients seldom express their concerns and emotions directly and spontaneously, but instead give indirect cues that something is worrying them (Zimmermann et al., 2007; 2011; Del Piccolo et al., 2012). Furthermore, closed questions themselves automatically block behaviours by the expectation that when a question is asked a response will be forthcoming, rather than free flow of communication.

In this paper we use a conversational analysis (CA) approach to examine the interactions between interviewers and older people during administration of the Philadelphia Geriatric Morale Scale (PGMS). We address five conversation events: institutional talk; rephrasing of questionnaire questions; missed cues; and the impact of closed questions in blocking rapport 
between the interviewer and responder and relate this to interactive engagement with older people.

\section{Methods}

The parent study, from which this paper is drawn, explored resident outcome measures that could help evaluate how living in care home facilities affects residents" quality of life (QoL). Three questionnaires were used; the World Health Organisation Quality of Life (WHOQOLBREF) (US and UK versions) instrument measuring the physical, psychological, social, and environmental domains; the WHOQOL Spirituality, Religiousness and Personal Beliefs (SRPB) instrument measured on an importance scale, which augments the assessment of the spirituality and personal beliefs domain; and the Philadelphia Geriatric Centre Morale Scale (PGCMS) measuring dimensions of attitudes towards ageing, agitation and lonely dissatisfaction. The parent study has been conducted with residents in care home facilities in Seattle, USA, $(n=49)$ West Sussex and Surrey in the UK $(n=39)$ and in the lower North Island, New Zealand (NZ) (n=37). Almost all residents requested assistance in completing the questionnaires. This was mainly due to physical limitations such as arthritis, Parkinson"es disease, physical effects of stroke, hearing or vision loss, or simply resident preference. It is common for old people to request researchers to administer questionnaires because of frailty in writing, unfamiliarity with written questionnaires, general physical and mental frailty and respondent fatigue, or using the situation to give them the opportunity to engage with others (Coast, Peters, Richards, \& Gunnell, 1998; Isaksson, Santamäki-Fischer, Nygren, Lundman, \& Åström, 2007).

Trained interviewers conducted interviews with residents in their care home room. While following the structured questionnaires as the interview guide, all interviewers noted 
considerable amounts of ,story telling“ or elaboration in resident responses. We began to pay particular attention to the conversations that occurred during administration of the questionnaires in care facilities in West Sussex, UK and notes taken revealed insights into events and experiences that impact on the QoL of the residents that were not being captured in the questionnaire data or in the accompanying field notes. Consequently we decided to audio-record the administration of the questionnaires. The addition of the audio recordings of the UK $(n=8)$ and NZ $(n=36)$ allowed us to make comparisons between transcribed conversation and responses recorded on the questionnaires. This paper focuses transcripts of The Philadelphia Geriatric Centre Morale Scale in a care home facility in NZ to explore two selected interactions between interviewer and the resident using a CA approach. Ethical approval for the questionnaire survey research was attained from the University of Washington IRB in the USA and the University of Surrey Ethical Review Board in the UK and Central Ethics for Health and Disability in NZ. Supplementary ethical approval was gained to include audio-recorded conversations during administration of the questionnaires in Surrey, UK and in NZ.

Inclusion criteria for participation in the study were: the resident was judged by staff (normally the manager of the care home facility) as capable of participation; English speaking; and must have been resident in the home for at least one year. Care home facility managers initially approached residents and provided them with the information letter about the research. The resident (on some occasions in consultation with families or significant others) made the decision to participate in the study. Prior to administering the questionnaires the resident signed a consent form, including consent to have the administration of the questionnaire audio-recorded. The questionnaires were anonymized, and any information about each participant in relation to the care home facility, address or any identifying code 
removed so that they could not be recognised. Quantitative data were analysed using SPSS software to explore the reliability of the scales and to explore comparisons between Seattle, USA, UK and NZ care facility populations (Results of the parent study have been reported elsewhere or are available from the authors and publication forthcoming).

\section{Conversational Analysis}

Conversational analysis (CA) is an alternative methodology to be considered to augment the standardised questionnaire. CA approaches language as a form of social action in the sense that it is integral to social interaction where the organisation of taking turns to talk is fundamental to conversation or other speech exchange systems (Sacks et al. 1974, 1978). When using CA particular emphasis is placed on pattern and context for the understanding of communicative exchanges during conversation (Sacks et al. 1974, 1978). In CA audiorecorded transcriptions are analysed for a broad range of speech exchange systems, or spoken interaction, collectively known as „talk-in-interaction“e (Sacks et al. 1974, 1978; Markee, 2007). Houtkoop-Steenstra (2000) has long been considered an authority on using this methodology in the analysis of questionnaire data, with a specific interest in the interactions between interviewer and interviewee during the administration of survey questionnaires and in particular, interview conversations that occurred within institutions.

Following the CA methodology, this paper primarily focuses on a section of one transcription of the PGCMS questionnaire and discusses the impact on the interaction with the older person during the process of closely following a questionnaire protocol. The questionnaire session under discussion was administered by one of our team early in her involvement with the research. The interviewer was a very experienced nurse but was a relatively novice researcher and was determined that the questionnaires were administered in keeping with a 
standardised approach to ensure collection of valid and reliable data. The second extract was conducted by an experienced researcher/interviewer and provides points of comparison for our discussion.

\section{Findings and discussion}

In initial analysis of the Philadelphia Geriatric Center Morale Scale (PGCMS) questionnaire, where we compared the audio-recorded responses with the questionnaire results, we found that the response to the question; "I have a lot to be sad about", was often the first opportunity offered to residents to express or reflect on bereavement experiences. Both of the respondents from which these extracts have been taken scored low on the PGCMS questionnaire (4 and 6 respectively; Mean for NZ=11.8; $\mathrm{SD}=3.4$ ) indicating that these residents may have some degree of depressed mood or low morale.

CA notation meanings are provided in Box 1.

\section{Box 1: CA Symbols}

\begin{tabular}{|ll|}
\hline$?$ & appeal (final) \\
$?$ & appeal (continuing) \\
$($ h) & laugher in speech \\
underlining & indicates emphasis \\
$\uparrow$ & marked rise in voice pitch \\
$\mathfrak{f}$ & indicates speaker was smiling while speaking \\
$\circ$ & quiet soft speech \\
$\circ \circ$ & whisper \\
$(0.4)$ & pause timed (4 tenths of a second) \\
$()$. & micropause, hearable but too short to measure
\end{tabular}

Extract \#1

1. I: As you get older you are less useful.

2. R: Awh (h-ironic) yes (h-ironic)

3. I: I sometimes worry so much that I can't sleep. 
4. R: No

6. would be.

7. R: ${ }^{\circ}$ Worse $^{\circ}$

8. I: I sometimes feel that life isn't worth living.

9. R: ${ }^{\circ}$ Yes $^{\circ}$

10. I: I am as happy now as when I was younger.

11. R: $(.)^{\circ \circ} \mathrm{No}^{\circ \circ}$

12. I: I have a lot to be sad about. (0.4) I have a lot to be sad about. Yes or no? Do you

13. have much to be sad about?

14. R: ${ }^{\circ}$ He's dead ${ }^{\circ}$ [YES MARKED ON QUESTIONNAIRE]

15. I: ${ }^{\circ}$ Yes ${ }^{\circ}[$ INTERVIEWER TO SELF] (0.2) I am afraid of a lot of things.

16. R: ${ }^{\circ}{ }^{\circ} \mathrm{No}^{\circ \circ}$

17. I: I get mad more than I used to.

18. R: (h-ironic) What's the use

19. I: So is that a no?

20. R: Awh I suppose so [NO MARKED ON QUESTIONNAIRE]

In this extract the interviewer responded to a perceived positive response using a high-grade assessment, an enthusiastic, “Well that's good" (line 5). In this case the interviewer was not using a pivotal particle marker like "ok" as a means of closing off each question on receiving the answer to the previous one (Anaki et al., 2000). The response to the question on sleep explicitly signalled the previous material as "having been successfully, indeed praiseworthily, completed" (Anaki et al., 2000:246) and signals a closure that allowed the interviewer to move immediately to the next question. There is no hesitation or pause at this point, however ironic laughter from the resident in response to "As you get older you are less useful" (line 1) could be deemed non-closure of the line of inquiry by the resident, as irony implies a deliberate contrast between apparent and intended meaning of her "yes" response to the question. At this point, if this had been an open-ended interview or conversation, the researcher would have explored the response.

The next question introduces a particularly sensitive line of enquiry "I sometimes feel that life isn't worth living" (line 8) to which the resident responds "yes" (line 9). The response and tone of the resident should have signalled concerns about her emotional and possibly her mental state, however these were left unacknowledged and unexplored to the extent that her 
response to the next question "I am as happy now as when I was younger" is a whispered "no" (lines 10,11). This reflects concerns that have been raised that the questionnaire could be termed as not fit for the situation and type of respondents for which it was being used (Houtkoop-Steenstra \& Antaki, 1997, Houtkoop-Steenstra, 2000) as they offer the opportunity for the resident to reflect on their happiness (or not) which is left unexplored and unsupported.

Both the interviewer and resident had not paused in responding to each other until the question asked (lines 12,13). At this question the resident pauses for the first time. "I have a lot to be sad about. Yes or no?" (pause). The interviewer does not allow the pause to continue for long. She rephrases the question with "Do you have much to be sad about?" This is an example of revising and repairing questionnaire items (Houtkoop-Steenstra \& Antaki, 1997; Houtkoop-Steenstra, 2000). However the question again raises concerns about the appropriateness of initiating a conversation with older people who may be experiencing bereavement and loss. Using questionnaires may shut down any potential for meaningful engagement and emotional expression and in the extract we present the bereavement experiences of the older person was denied and disacknowledged. In this case the interviewer recorded YES on the questionnaire in response to the resident saying " $H e$ 's dead" to the question "Do you have much to be sad about?" However the YES recorded, based on the resident"s response, would appear to be an accurate recording despite the lack of investigation by the interviewer to the response. This same section of data (lines 12-14) we believe also demonstrated an example of a ,missed cue ${ }^{e e}$. 


\section{Missing cues}

We posit that focusing on the administration of the questionnaire and a desire to get ,valide data caused the interviewer to be detached from the emotions and feelings of the older person. On line 14 the response to the question, "Do you have much to be sad about?" after a pause, is simply "He's dead". The interviewer had no recollection of the resident making this statement. What occurs in the interaction is that the interviewer does not „listen ${ }^{\text {ee }}$ to the responses and when cues are missed the likelihood of information (and emotion) not being expressed, captured or qualified increases (Maguire, Faulkner, Booth, Elliott, \& Hillier, 1996; Maguire \& Pitceathly, 2002). When examining the transcripts she was horrified at the enormity of missing a cue that indicated a possible depth of sadness that the resident may have been experiencing about the death of the husband. The despondency of the resident becomes even more evident as the interview continued. We propose that this occurred due to the non-engagement of the interviewer who was focused on completing the questionnaire and was not „listening" to the responses. The interviewer marks YES on the questionnaire, murmuring "yes" to herself and with minimal pause asks the next question, "I am afraid of a lot of things" (line 15) the response of the resident is barely audible and on line 18 she expresses her feelings quite explicitly "What's the use” with ironic laughter. This response appears to have as much to do with her responses to the interactions occurring within the „conversation" as to do with her experience of loss regarding her husband.

In this extract the interviewer, although a healthcare professional, did not attempt to clarify or investigate a potentially significant emotional situation at the time. We further conclude that it was possible that the sadness of the response was overwhelming and not able to be addressed by the interviewer, even though she had no recollection of the response by the resident. The focus of research on responding to patient cues has been predominantly 
conducted in the field of communication with cancer or palliative care patients. In these fields it has been found that patients seldom express their concerns and emotions directly and spontaneously, but instead give indirect cues that something is worrying them. Research has demonstrated that both doctors and nurses have difficulty both eliciting and responding to cues that may heighten their own emotional responses, or to emotions displayed by their patients, and use blocking behaviours, primarily to protect themselves (Uitterhoeve et al., 2008; Uitterhoeve et al., 2009; Mjaaland, Finset, Jensen, \& Gulbrandsen, 2011).

\section{Impact of closed questions and blocking behaviours}

During the study, on initial meeting, residents were generally animated and keen to talk. In a number of cases however the resident became more and more despondent and less interested in the questions or the encounter as the questioning progressed that required YES or NO answers, or responses to a Likert Scale. One of the reasons for this despondency could be attributed to the nature of the questioning style i.e. use of closed questions. The question format on the questionnaire discussed in this paper was that of closed questions which are typically used in questionnaires and are limited to a predetermined set of responses (Houtkoop-Steenstra, 2000). As seen in the closed question example cited earlier (lines 1220) elicited a YES/NO response with little else. It was noted that the impact of asking closed questions was to „shut down" the older person quite quickly blocking interaction, consequently very little communication spoken or otherwise takes place.

Extract \#2 is presented here as a comparison piece.

1. I: Things keep getting worse as I get older? (.04) It's just a yes or no answer.

2. R: (.4) I'd have to say yes wouldn't I? [WORSE MARKED ON QUESTIONNAIRE].

3. I: (.2) Because it isn't what you expected to happen is it? [REFERRING TO ILLNESS]

4. R: (.2) No.

5. I: (.) No

6. R: (.) But (.) no that's true (.) that's true 
7. R: (.2) You could enter that but that's not that they want (.) um

8. I: Well you don't have to (.) it can be yes or no or somewhere in the middle because

9. R: Yes, oh well just put yes.

10. I: (.2) It's something you don't think about.

11. R: Oh no $\uparrow$ I've thought about it, I never thought that I would be old and get sick.

12. I: No.

13. R: A friend came yesterday you know. Joe

14. I: Yeah

15. R: And he said he never, you know, that he'd see me like this, you know

16. I: $M m$

17. R: because I'm usually full of beans running around

18. I: Yeah

19. R: and I won't be doing that again no matter what unless they come up with some magical

20. R: cure

21. I: Yeah

22. R: but that ain't going to happen

23. I: No probably not (.) not in the short term (.) no.

24. $\mathrm{R}$ : but then again (.2)

25. I: Yeah

26. R: They said one thing you can be sure of it will get worse.

27. I: (.) Yeah

28. R: (.) Yeah

29. I: (.) Yeah. At least they've talked about it really well with you haven't they.

30. R: Yes.

31. I: About the illness and how it will progress and [OVERLAPPING]

32. R: and how they could help

33. I: Yeah

This extract presents a similar question from the PGCMS to illustrate the researcher/resident interaction. In the extract the interviewer uses a more conversational approach (Suchman \& Jordan, 1990; Schober \& Conrad, 1997; Conrad \& Schober, 2000; Isaksson et. al., 2007)) when interacting with the resident. The interviewer also draws on information gained earlier in the meeting about a recent diagnosis of an illness the resident was experiencing (line 3), as the response to the question, "Things keep getting worse as I get older?" was clearly related to this. Lines 4 to 10 demonstrate the interviewer allowing time for the resident to contemplate and reflect on the reference to this illness. The interviewer also offers a potential „middle ground“ as a response. This example of revising and repairing questionnaire items (Houtkoop-Steenstra \& Antaki, 1997; Houtkoop-Steenstra, 2000) would potentially lead to an invalid or incomplete questionnaire as the responses required were YES or NO. Based on the conversational approach to administering the questionnaire the interviewer was 
acknowledging that yes or no may not adequately express the position of the resident about getting older. This offer is not taken up by the resident, who settles on "oh well just put yes", which is however not a firm affirmative.

At line 10 the interviewer makes a statement suggesting that the resident has not reflected on the impact of her illness. This is immediately met with a response that indicates that the illness was very close to mind and there are no contemplative pauses between lines 11 to 22 where the resident reveals that she understands the impact of her illness and that there is no cure. On line 23 the interviewer acknowledges that there is no cure "in the short term" which allows the resident to contemplatively express hope "but then again", (line 24) and the interaction continues with a contemplative exchange of yeah, pause yeah pause. This interaction allows the interviewer to again refer to the resident"s illness offering the resident the opportunity to continue the discussion and to extend this to include further exploration of what engagements the resident may have had with the medical team regarding her future.

Suchman and Jordan (1990) argue that the standardised procedures used when conducting a survey interview suppresses crucial elements of ordinary conversation, and, in the first extract presented here, emotional expression. Their seminal paper is widely cited in debates on the validity of survey data where standardisation is understood to be that which identifies the interview process as a scientific procedure. However they show that the survey interview is "fundamentally an interactional event" (241) and propose taking a collaborative approach to the problem that; "would allow the kinds of interactional exchanges between interviewer and respondent necessary to ensure standardized interpretations, without introducing interviewer bias" (232) which is demonstrated in extract 2. In taking this approach the interviewer would be allowed to discuss the meaning of questions, offer clarification and elaboration, and to 
engage in a "limited form" of discussion and interpretation. Thus they would also be empowered to respond to emotional expressions that may arise, particularly when administering questionnaires to the respondent group under discussion in this paper. Whilst the first extract is an extreme example our data demonstrates multiple occasions of rephrasing questions, missed cues, and evidence of closed questions shutting down residents.

\section{Conclusion}

QoL and other questionnaires are frequently used in research undertaken in aged care. There has been little study in this field that considers the impact of using questionnaires in research with older people. Using a CA approach we have shown how institutional talk, missing cues, and the use of closed questions and blocking behaviours are inhibitors and barriers to communication with older people. Rigid adherence to questionnaire protocol may deny and disacknowledge emotional expression and consequently be detrimental to the older person ${ }^{\text {ee }} \mathrm{s}$ psychological health. The approach disables the interviewer from interacting and engaging in a meaningful conversation and creating a therapeutic encounter. Furthermore, the validity of the data obtained by this method is potentially undermined by the constraint this places on interaction between the interviewer and respondent. Conrad and Schober (2000) have shown that conversational (to collect survey data) take three times as long to conduct as stanadarised interviews. However, in regard to the population under discussion and based on our analysis and experiences of conducting this research we support the recommendations that both a collaborative (Suchman \& Jordan, 1990) and supportive (Isaksson et. al., 2007) approach, allowing an interactional exchange between interviewer and respondent, be used when administering questionnaires to older people in care home facilities. This approach rejects the "strategy that mistakes sameness of words for stability of meanings" (Suchman \& Jordan, 1990:233). We agree with Suchman and Jordan that stability of meaning in administering 
questionnaires to older people depends on an engaged conversational interaction. This allows collecting systematic quantitative data, enhances the accuracy of the data collected by focusing on the meaning of the questions, and concurrently results in a meaningful conversation that could possibly include a referral for further attention if such a need was identified.

\section{References}

Antaki, C., Houtkoop-Steenstra, H. \& Rapley, M. 2000. "Brilliant. Next Question. ..": HighGrade Assessment Sequences in the Completion of Interactional Units. Research on Language \& Social Interaction 33(3): 235-262. doi.org/10.1207/S15327973RLSI3303_1

Coast, J., Peters, T.J., Richards, S.H. \& Gunnell, D.J. 1998. Use of the EuroQoL among elderly acute care patients. Quality of Life Research 7(1): 1-10.

Conrad, F.G., Scober, M.F. 2000. Clarifying question meaning in a household telephone survey. Public Opinion Quarterly 64, 1-28.

Del Piccolo, L., Goss, C. \& Bergvik, S. 2006. The fourth meeting of the Verona network on sequence analysis ,.,consensus finding on the appropriateness of provider responses to patient cues and concerns ${ }^{\text {ecee }}$. Patient Education and Counseling 61:473-475.

Del Piccolo, L., Mazzi, M. A., Goss, C., Rimondini, M. \& Zimmermann, C. 2012. How emotions emerge and are dealt with in first diagnostic consultations in psychiatry. Patient Education and Counseling 88, 29-35. Retrieved from www.elsevier.com/locate/pateducou

Ernst, M.O. \& Bülthoff, H.H. 2004. Merging the senses into a robust percept. Trends in Cognitive Sciences 8(4), 162-169. doi: 10.1016/j.tics.2004.02.002

Fowler, F.J. \& Mangione, T.W. 1990. Standardized survey interviewing: Minimizing interviewer related error. Newbury Park, CA: Sage.

Houtkoop-Steenstra, H. 1996. Probing behaviour of interviewers in the standardised semiopen research interview. Quality \& Quantity 30: 205-230.

Houtkoop-Steenstra, H. \& Antaki, C. 1997. Creating happy people by asking yes-no questions. Research on Language \& Social Interaction 30(4): 285-313.

http://dx.doi.org/10.1207/s15327973rlsi3004_2

Houtkoop-Steenstra, H. 2000. Interaction and the standardized survey: The living questionnaire. Cambridge, England: Cambridge University Press.

Isaksson, U., Santamäki-Fischer, R., Nygren, B., Lundman, B. \& Åström, S. 2007. Supporting the very old when completing a questionnaire: Risking bias or gaining valid results? Research on Aging 29(6): 576-589. DOI: 10.1177/0164027507305924 
Maguire, P., Faulkner, A., Booth, K., Elliott, C. \& Hillier, V. 1996. Helping cancer patients disclose their concerns. European Journal of Cancer, 32A: 78-81.

Maguire, P. \& Pitceathly, C. 2002. Key communication skills and how to acquire them. British Medical Journal 325: 697-700.

Markee, N. 2007. Conversation analysis: Issues and problems. In Cummins, J., Davison, C. (eds) International Handbook of English Language Teaching. Springer: New York. Pp10171032.

Mjaaland, T.A., Finset, A., Jensen, B.F. \& Gulbrandsen, P. 2011. Physicians ${ }^{\text {ee }}$ responses to patients ${ }^{\text {ee }}$ expressions of negative emotions in hospital consultations: A video-based observational study. Patient Education and Counseling 84: 332-337.

doi:10.1016/j.pec.2011.02.001

Sacks, H., Schegloff, E. A., \& Jefferson, G. (1974). A simplest systematics for the organization of turn-taking for conversation. Language, 50, 696-735.

Sacks, H., Schegloff, E. A., \& Jefferson, G. (1978). A simplest systematics for the organization of turn-taking for conversation. In: Jim Schenkein (ed) Studies in the organization of conversational interaction. New York Academic Press. 1-55.

Schaeffer, N.C. \& Maynard, D.W. 2002. Occasions for Intervention: Interactional Resources for Comprehension in Standardized Survey Interviews. In: Maynard, D. W., HoutkoopSteenstra, H., Schaeffe, N.C. and van der Zouwen, J. (eds). Standardization and Tacit Knowledge: Interaction and Practice in the Survey Interview. New York: Wiley. Pp. 261-280

Schober, M.F. \& Conrad, F.G. 1997. Does conversational interviewing reduce measurement error? Public Opinion Quarterly 61: 576-602.

Suchman, L. \& Jordan, B. 1990. Interactional trouble in face-to face interviews. Journal of the American Statistical Association 85(409): 232-241.

Uitterhoeve, R., de Leeuw, J., Bensing, J., Heaven, C., Borm, G., deMulder P. \& van Achterberg, T. 2008. Cue-responding behaviours of oncology nurses in video-simulated interviews. Journal of Advanced Nursing 61(1): 71-80. doi: 10.1111/j.13652648.2007.04467.x

Uitterhoeve, R., Bensing, J., Dilven, E., Donders, R., deMulder, P. \& van Achterberg, T. 2009. Nurse-patient communication in cancer care: does responding to patient,,s cues predict patient satisfaction with communication. Psycho-Oncology 18:1060-1068. DOI: 10.1002/pon. 1434

van „t Hof, S. 2006. From Text to Talk: Answers and their uptake in standardised survey interviews. Netherlands Graduate School of Linguistics/Landelijke (LOT): Utrecht, The Netherlands. http://www.lotpublications.nl/publish/articles/001965/bookpart.pdf (Accessed 20/03/2014) 
Viterna, J.S. \& Maynard, D.W. 2002. How Uniform Is Standardization? Variation Within and Across Survey Research Centers Regarding Protocols for Interviewing. In: Maynard, D. W., Houtkoop-Steenstra, H., Schaeffe, N.C. and van der Zouwen, J. (eds). Standardization and Tacit Knowledge: Interaction and Practice in the Survey Interview. New York: Wiley. Pp 365-397

Zimmermann, C., Del Piccolo, L. \& Finset, A. 2007. Cues and concerns by patients in medical consultations: A literature review. Psychological Bulletin 133(3): 438-463. doi: 10.1037/0033-2909.133.3.438.

Zimmermann, C., Del Piccolo, L., Bensing, J., Bergvik, S., De Haes, H., Eide, H., Fletcher, I., Goss, C., Heaven, C., Humphris, G., Kim, Y., Langewitz, W., Meeuwesen, L., Nuebling, M., Rimondini, M., Salmon, P., van Dulmen, S., Wissowm, L., Zandbelt, L., \& Finset, A. 2011. Coding patient emotional cues and concerns in medical consultations: The Verona coding definitions of emotional sequences (VR-CoDES). Patient Education Counselling 82(2): 141-148. doi:10.1016/j.pec.2010.03.017 